\title{
The Effect of Dietary Antioxidant Supplementation in Patients with Glaucoma
}

\author{
Noriko Himori (1) \\ Maki Inoue Yanagimachi' \\ Kazuko Omodaka ${ }^{1,2}$ \\ Yukihiro Shiga' \\ Satoru Tsuda' \\ Hiroshi Kunikata ${ }^{1,3}$ \\ Toru Nakazawa ${ }^{\text {-4 }}$ \\ 'Department of Ophthalmology, Tohoku \\ University Graduate School of Medicine, \\ Sendai, Japan; ${ }^{2}$ Department of \\ Ophthalmic Imaging and Information \\ Analytics, Tohoku University Graduate \\ School of Medicine, Sendai, Japan; \\ ${ }^{3}$ Department of Retinal Disease Control, \\ Tohoku University Graduate School of \\ Medicine, Sendai, Japan; ${ }^{4}$ Department of \\ Advanced Ophthalmic Medicine, Tohoku \\ University Graduate School of Medicine, \\ Sendai, Japan
}

Correspondence: Toru Nakazawa Department of Ophthalmology, Tohoku University Graduate School of Medicine, I-I Seiryo-cho Aoba-ku, Sendai, Miyagi, 980-8574, Japan

Tel $+8 I-22-7 \mid 7-7294$

Fax +8I-22-7I7-7298

Email ntoru@oph.med.tohoku.ac.jp
Purpose: Oxidative stress may be a risk factor for glaucoma, and many previous reports have suggested that antioxidants could be a promising treatment. Here, we investigated the effects of a novel supplement containing three food-derived antioxidants (hesperidin, crocetin, and Tamarindus indica) on markers of oxidative stress in patients with glaucoma.

Patients and Methods: This study had a prospective, single arm design. Thirty Japanese glaucoma patients were recruited and given 4 tablets with ample water twice a day for 8 weeks. The treatment was stopped, and the subjects were followed for an additional 8 weeks. We measured biological antioxidant potential (BAP) with a free radical analyzer. We also measured urinary 8-hydroxy-2'-deoxyguanosine $(8-\mathrm{OHdG}$; a marker of oxidative DNA damage). Clinical laboratory data were measured in venous blood samples. Clinical parameters were also recorded. Comparisons used a one-way analysis of variance (ANOVA) followed by Dunnett's test.

Results: The 8-OHdG level was not reduced. We also divided the patients into groups with high or low oxidative stress. In patients with relatively high oxidative stress, the 8-OHdG level was significantly reduced at weeks $4,8,12$, and $16(P<0.001, P<0.01, P<0.01, P<$ $0.01)$, and BAP was significantly elevated at weeks 8 and $12(P=0.03, P=0.04)$. In patients with relatively low oxidative stress, the $8-\mathrm{OHdG}$ level was not significantly reduced during supplement intake but was significantly elevated at weeks 12 and $16(P=0.03, P=0.04)$, while BAP was not significantly elevated.

Conclusion: An 8-week oral course of antioxidant supplementation was effective in patients with a high oxidative stress level. Dietary supplementation could hold promise in the treatment of systemic oxidative stress-related diseases.

Keywords: oxidative stress, supplement, glaucoma, 8-OHdG

\section{Introduction}

Oxidative stress in the body is normally managed by balancing the production of reactive oxygen species (ROS) with the activation of a variety of antioxidative cellular mechanisms. The efficacy of these mechanisms is critical, because if they are impaired, oxidative damage can continue even when overall oxidative stress is within normal levels. Oxidative damage to proteins, DNA, and lipids contributes to many age-related neurodegenerative diseases, as well as age-related macular degeneration, diabetic retinopathy, cataract, and glaucoma. ${ }^{1-5}$ The World Health Organization estimates that among the 37 million people with blindness worldwide, $12 \%$ have lost their sight due to glaucoma. ${ }^{6}$

Treatment for glaucoma most commonly involves therapy to reduce intraocular pressure (IOP). However, disease progression persists in some patients even after 
IOP is successfully reduced. This has led to past investigations of possible non-IOP risk factors for glaucoma and its key underlying pathomechanism, retinal ganglion cell (RGC) degeneration. Previously reported risk factors include genetics, ${ }^{7}$ vascular dysregulation, ${ }^{8}$ mitochondrial dysfunction, ${ }^{9}$ and in particular, oxidative stress. ${ }^{2}$ Oxidative stress has three key effects in glaucoma. First, it raises IOP by altering the trabecular meshwork and impairing aqueous humor outflow. ${ }^{10,11}$ Second, it disrupts autoregulation of blood flow to the optic nerve by altering the vessels that feed it. ${ }^{12,13}$ Finally, patients with a low antioxidant level in the eye are also susceptible to systemic oxidative stress, which can induce RGC death, as we have previously reported. ${ }^{14,15}$ Harris et al revealed that one month of oral supplementation with an antioxidant increased biomarkers of blood flow in the eyes of patients with open-angle glaucoma (OAG). ${ }^{16}$ Park et al found that a four-week course of daily administration of $160 \mathrm{mg}$ of Gingko biloba extract led to a significant improvement in retinal blood flow volume and velocity in 15 patients with normaltension glaucoma (NTG). ${ }^{17}$ In vivo experiments using animal models of glaucoma (based on apoptosis signal regulating kinase 1 , valproic acid, $\mathrm{N}$-acetylcysteine, spermidine, and coenzyme Q10) have showed that administering antioxidants had a neuroprotective effect. ${ }^{18}$ These research suggest that antioxidants are a potential candidate for glaucoma therapy.

Here, we gave a daily dietary antioxidant supplement over 8 weeks to patients with glaucoma. The supplement was developed and first reported by Maekawa et al, who identified the components of the supplement through screening and reported that three food-derived compounds, hesperidin, Tamarindus indica, and crocetin, had a protective effect in a primary culture of retinal cells under oxidative stress. ${ }^{19}$ Moreover, hesperidin reduced apoptosis, oxidative stress and inflammation and had a neuroprotective effect in a mouse glaucoma model. We believe that the findings reported here will shed light on the potential of new treatments for glaucoma based on antioxidant supplementation.

\section{Patient and Methods}

\section{Study Design}

This study had a prospective, single arm design. Patients with previously diagnosed NTG were recruited at Tohoku University Hospital between March 2018 and
October 2019; all patients were Japanese and all were followed for 16 weeks.

The sample size was based on a preliminary study (unpublished) that used the same method as the present study to measure average BAP before and after supplement intake. The preliminary study showed that BAP increased by 98.6 (standard deviation: 157.9). When we set the $\alpha$ error at 0.05 and the power at 0.8 , we found that the number of subjects to obtain statistically significant results in a comparative study was 23 . Thus, we set the number of subjects at 30 in anticipation of dropouts.

This study adhered to the Declaration of Helsinki and Clinical Trials Act and was approved by the Clinical Research Review Board of Tohoku University (study 2019-6-068), which is certified by the Japanese Ministry of Health, Labor and Welfare. The trial was registered with the UMIN clinical trial registry, number 000032050. Written informed consent was obtained from all subjects before the start of the study.

\section{Participants}

Inclusion criteria for participation were as follows: overall good health, age between 30 and 75 years; NTG confirmed in one or both eyes by a glaucoma specialist; a biological antioxidant potential (BAP) level between 1600 and 2200 $\mathrm{nmol} / \mathrm{L}$; and body mass index less than $26 \mathrm{~kg} / \mathrm{m}^{2}$. Exclusion criteria were as follows: hyperopia (above $+3 \mathrm{D}$ ); high myopia (below $-8 \mathrm{D}$ ); any type of secondary glaucoma; high IOP (> $16 \mathrm{mmHg}$ ) despite glaucoma medication; and severe systemic disease, including cancer, hyperthyroidism, and autoimmune disease. No patients used exogenous hormones. Pregnant women, or women planning pregnancy during the study period, were also excluded. Candidates were asked to refrain from the use of vitamin or carotenoid supplements for 2 weeks before the study. If a patient had diabetes, hyperlipidemia, hypertension, or a current smoking habit, it was recorded.

Figure 1 shows an overview of the study design. Participants were examined 5 times, at four-week intervals. At the examination, we measured clinical parameters, confirmed compliance with the study protocol of twicedaily use of the supplement, and recorded any adverse events.

\section{Study Intervention}

Maekawa et al previously reported 12 candidate neuroprotective compounds. ${ }^{19}$ Based on these results, we developed the novel supplement used in this study, which contained 


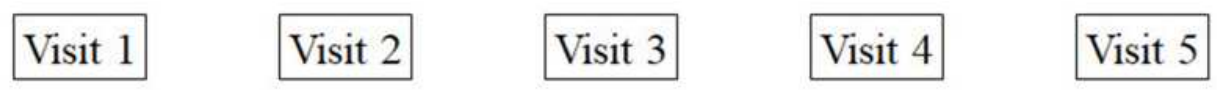

Entry at screening test $(\mathrm{n}=30)$

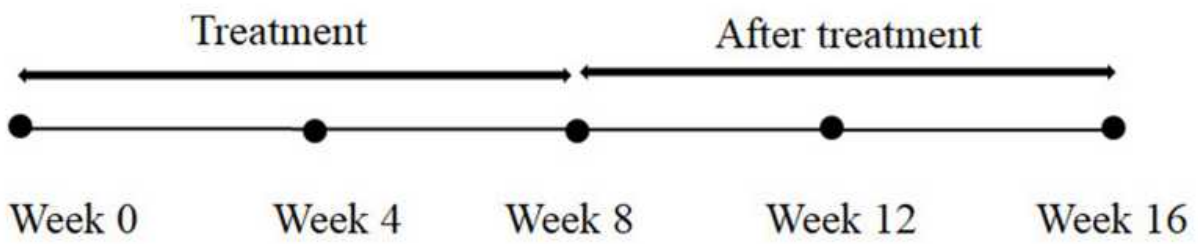

Figure I Overview of study design.

the following ingredients in each daily dose of 4 tablets: hesperidin (50 mg/4 tablets), crocetin ( $7.5 \mathrm{mg} / 4$ tablets), and Tamarindus indica (25 mg/4 tablets). The supplement was manufactured and supplied by Wakamoto Pharmaceutical Co. Ltd (Tokyo, Japan). The candidates took 2 tablets with ample water twice a day (ie, 4 tablets/day) for 8 weeks, and no supplementation for 8 weeks. Any unused supplements were returned for a pill count at the 8-week visit.

\section{Measurements}

A complete ophthalmic examination of all patients was performed by a glaucoma specialist. This comprised measurement of best-corrected visual acuity (recorded as the logarithm of the minimum angle of resolution) examinations with slit-lamp biomicroscopy and funduscopy, and evaluation of the optic disc with a 90-diopter lens. The patients with glaucoma also underwent measurement of mean deviation (MD) with the Humphrey field analyzer (HFA). If both eyes had glaucoma, the analysis included the worse-MD eye.

\section{Clinical Laboratory Measurements}

Samples of blood were stored in containers with EDTA at weeks 0 and 8 . We confirmed supplement safety based on clinical laboratory measurements. Plasma samples were stored at $-80^{\circ} \mathrm{C}$ for later biochemical analysis. Serum samples from the screening visit were sent to LSI Medience Co. (Tokyo, Japan), analyzed for AST, ALT, $\gamma$ GTP, total bilirubin, total protein, albumin, high-density lipoprotein cholesterol, low-density lipoprotein cholesterol, total cholesterol, triglycerides, creatinine, blood urea nitrogen, uric acid, glucose, $\mathrm{HbA} 1_{C}$, white and red blood cell count, platelet count, hematocrit, and hemoglobin.

\section{Blood Sampling and Oxidative Stress Measurement}

Serum samples were evaluated for oxidative stress levels and anti-oxidative potential with a free radical analyzer system (Free Carpe Diem, Wismerll Company Ltd., Tokyo, Japan). Blood samples were collected more than 3 hours after a subject ate. Total plasma antioxidant capacity in the samples was measured with the BAP test, which is based on the ability of a sample to reduce iron from its ferric $(\mathrm{Fe} 3+)$ to its ferrous $(\mathrm{Fe} 2+)$ state. The analyses were performed as previously reported. ${ }^{14,15}$

\section{Urinary Sampling}

Urinary levels of 8-oxo-2'-deoxyguanosine (8-OHdG), an oxidative stress marker, were measured with the commercially available ELISA kit (LSI Medience Co., Tokyo, Japan). Creatinine levels were also measured in urine samples. Urine samples were stored at $-4^{\circ} \mathrm{C}$.

\section{Statistical Analysis}

The significance of differences between groups was determined with the Mann-Whitney $U$-test and Fisher's exact test. Comparisons of groups used one-way analysis of variance (ANOVA) and Dunnett's test. All numerical findings are mean $\pm \mathrm{SD}$. Statistical significance was set at $\mathrm{P}<$ 0.05. SPSS version 23.0 (SPSS Inc., Chicago, IL, USA) was used for all analyses

\section{Results}

\section{Subjects}

This study enrolled 30 patients with NTG. Table 1 shows the demographic characteristics of the subjects. We also 
Table I Characteristics of Normal Tension Glaucoma Patients

\begin{tabular}{|c|c|c|c|c|}
\hline Demographics & All & 8-OHdG < 6.86 (ng/mg Creatinine) & 8-OHdG > 6.86 (ng/mg Creatinine) & $P$-value \\
\hline Number of patients & 30 & 19 & 11 & - \\
\hline Age (years) & $59.1 \pm 9.0$ & $60.7 \pm 8.5$ & $56.3 \pm 9.6$ & 0.23 \\
\hline Sex (male: female) & 15: 15 & 10: 9 & 5: 6 & $1.00 *$ \\
\hline Visual acuity (logMAR) & $0.11 \pm 0.37$ & $0.01 \pm 0.30$ & $0.08 \pm 0.34$ & 0.28 \\
\hline IOP (mmHg) & $11.03 \pm 2.25$ & $11.48 \pm 1.87$ & $11.58 \pm 1.97$ & 0.94 \\
\hline Mean deviation $(\mathrm{dB})$ & $-14.24 \pm 6.58$ & $-15.20 \pm 6.36$ & $-12.89 \pm 6.99$ & 0.49 \\
\hline BMI $\left(\mathrm{kg} / \mathrm{m}^{2}\right)$ & $22.34 \pm 2.15$ & $23.14 \pm 1.59$ & $20.95 \pm 2.35$ & 0.02 \\
\hline 8-OHdG (ng/mg creatinine) & $6.86 \pm 3.52$ & $4.69 \pm 1.41$ & $10.59 \pm 2.88$ & $<0.01$ \\
\hline $\mathrm{BAP}(\mu \mathrm{mol} / \mathrm{L})$ & $2022.37 \pm 139.94$ & $2039.56 \pm 110.53$ & $1992.64 \pm 182.40$ & 0.64 \\
\hline Diabetes (\%) & I (3.33) & $0(0.00)$ & I (9.09) & $0.37^{*}$ \\
\hline Hypertension (\%) & $4(13.33)$ & $4(21.05)$ & $0(0.00)$ & $0.27^{*}$ \\
\hline Current smoker (\%) & $6(20.00)$ & $3(15.79)$ & $3(27.27)$ & $0.64 *$ \\
\hline Hyperlipidemia (\%) & $6(20.00)$ & $5(26.32)$ & I (9.09) & $0.37^{*}$ \\
\hline
\end{tabular}

Notes: Unmarked $P$ values: Mann-Whitney $U$-test; ${ }^{*}$ Fisher exact test.

Abbreviations: 8-OHdG, 8-hydroxy-2'-deoxyguanosine; logMAR, logarithm of minimum angle of resolution; IOP, intraocular pressure; BMI, body mass index; BAP, biological antioxidant potential.

divided the patients into groups with high or low oxidative stress, defined by an 8 -OHdG level higher or lower than $6.86 \mathrm{ng} / \mathrm{mg}$ of creatinine, respectively, and performed separate analyses of these groups. There was a significant difference in body mass index and 8-OHdG between the two groups $(P=0.02, P<0.01)$. There were no supplement-related adverse events in any patient, and all blood tests were normal (data not shown).

\section{Oxidative Stress}

BAP was significantly elevated at weeks 8 and $16(P=$ $0.01, P=0.02$, respectively; Figure $2 \mathrm{~B})$. There were reductions in the 8 -OHdG level, but these did not reach statistical significance at any of the five time points (Figure 2A). In the high-oxidative stress group, the 8-OHdG level was significantly reduced at weeks 4,8 , 12 , and $16(P<0.001, P<0.01, P<0.01, P<0.01$, Figure $3 \mathrm{~B}$ ) and BAP was significantly elevated at weeks 8 and $12(P=0.03, P=0.04$, Figure $3 \mathrm{D})$. In the group with relatively lower oxidative stress, the 8 -OHdG level was not significantly reduced during supplement intake or after intake was stopped, but was significantly elevated at weeks 12 and $16(P=0.03, P=0.04$, Figure $3 \mathrm{~A})$; BAP was not significantly elevated (Figure $3 \mathrm{C}$ ).

\section{Discussion}

The results of this study are of particular interest for the treatment of patients with a low antioxidant level; we found that antioxidant supplementation may be an effective option for such patients. This is especially promising for the treatment of glaucoma, an age-related chronic neurodegenerative disease in which oxidative stress has been shown to play an important role.

The glaucoma patients in this study who had a relatively higher level of oxidative stress and took a daily antioxidant supplement showed an increase in BAP and a decrease in $8-\mathrm{OHdG}$, while the glaucoma patients with a relatively lower level of oxidative stress who took the supplement did not show an increase in BAP and showed a decrease in $8-\mathrm{OHdG}$. This suggests that our supplement was only effective in patients with a high oxidative stress level. Previously, Maekawa et al reported that three food-derived compounds, hesperidin, Tamarindus indica, and crocetin, had a protective effect in a primary culture of retinal cells under oxidative stress, and that hesperidin reduced apoptosis, oxidative stress and inflammation. Maekawa confirmed that hesperidin had these effects in vivo in mice, reducing oxidative stress and preventing RGC death caused by N-methyl- 
A

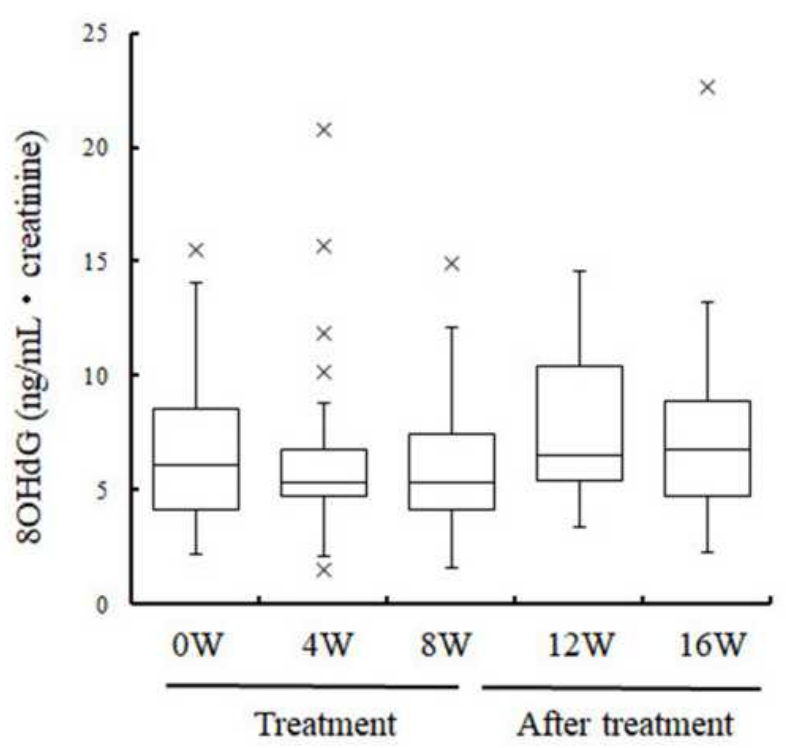

B

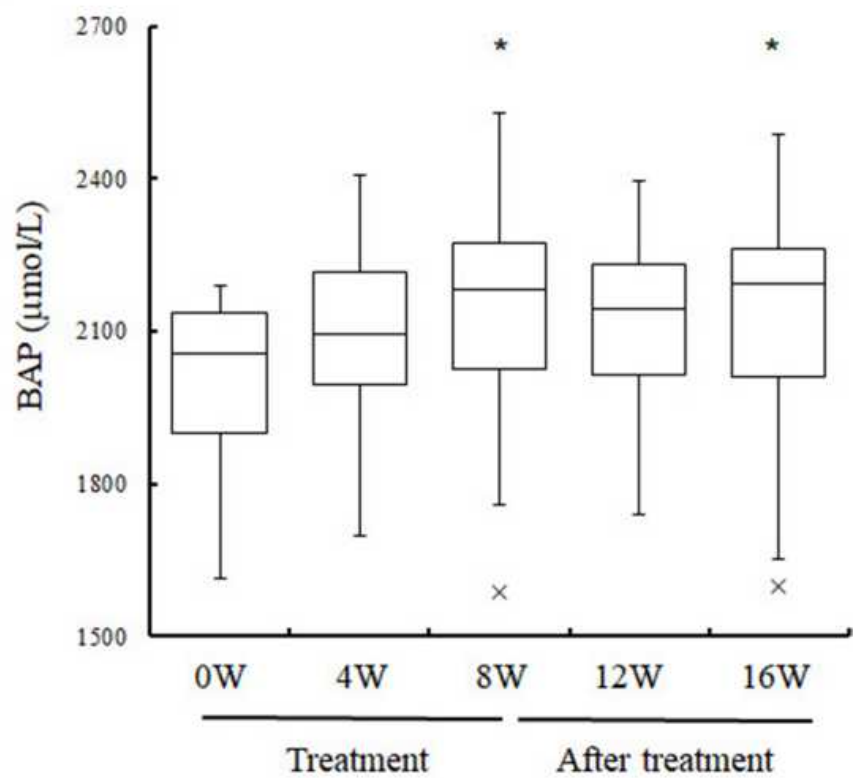

Figure 2 Comparison of findings before and after antioxidant treatment in patients with glaucoma. (A) There was no significant increase in 8-OHdG level. (B) BAP level significantly increased at weeks 8 and $16 . * p<0.05$.

D-aspartate (NMDA)-induced excitotoxicity. ${ }^{19}$ Tamarindus indica is indigenous to tropical Africa. It is high in tartaric acid, B vitamins, and minerals. It has antioxidant, ${ }^{20,21}$ anti-inflammatory, ${ }^{22}$ anti-diabetic, ${ }^{23}$ and anti-atherosclerotic effects. ${ }^{24}$ Crocetin is a natural apocarotenoid dicarboxylic acid that is found in the crocus flower and in Gardenia jasminoides. Crocetin has various effects, acting as an antioxidant ${ }^{25}$ and anti-inflammatory, ${ }^{26}$ and can inhibit the caspase pathway, preventing retinal damage induced by NMDA. ${ }^{27}$ These results suggest that these three food-derived compounds, used as a dietary supplement, might help reduce RGC degeneration in retinal disease.

A variety of antioxidant molecules can be found in human plasma, with the following compounds being most common: $\alpha$-tocopherol, $\beta$-carotene, albumin, ascorbic acid, bilirubin, catalase, ceruloplasmin, ferritin, glutathione peroxidase, lycopene, reduced glutathione, SOD, and uric acid. ${ }^{28}$ All these compounds can catalytically remove reactive species such as free radicals. After taking our novel supplement, the subjects in this study showed decreased oxidative stress and an increased antioxidative level. Interestingly, we found that patients showing a higher level of antioxidative activity also showed a lower level of oxidative stress. This is especially interesting for glaucoma, which can sometimes progress even with successful IOP-lowering treatment.
There is extensive clinical evidence that systemic oxidative stress contributes to glaucomatous optic neuropathy. Moreover, antioxidant therapy has shown promising results in animal- and human-based research. Antioxidant treatment can decrease the activation of NF$\kappa \beta$ and decrease the production of cytokines in the optic nerve and retina. ${ }^{29}$ In a rat glaucoma model, overexpression of thioredoxins can protect the RGCs. ${ }^{30}$ Higher green leafy vegetable intake is associated with a lower primary OAG risk. ${ }^{31}$ Lee et al found that Ginkgo biloba extract protected against the effects of glaucoma in some patients. $^{32}$ These results suggest that insufficient serum antioxidant proteins underlie the contribution of oxidative stress to glaucoma, indicating that antioxidants should have a neuroprotective effect. Our past research also showed that visual field damage was strongly correlated with systemic antioxidant levels in young, male patients with OAG. ${ }^{14}$ Antioxidant treatments might therefore be a useful therapeutic option to delay or prevent disease progression.

Limitations of this study included, first, that it lacked a placebo arm, which is needed to prove the efficacy of any supplement. Thus, we plan to conduct further studies with a randomized, doubled-blind, placebo-controlled design. Second, we observed a significant elevation in BAP after supplementation for eight weeks in the overall group of NTG patients (Figure 2B), and for four weeks in 
A

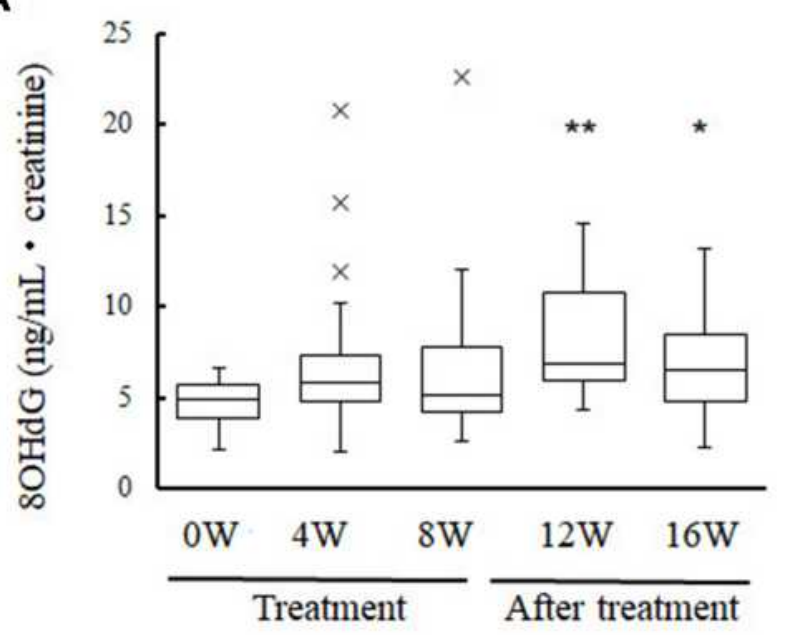

C

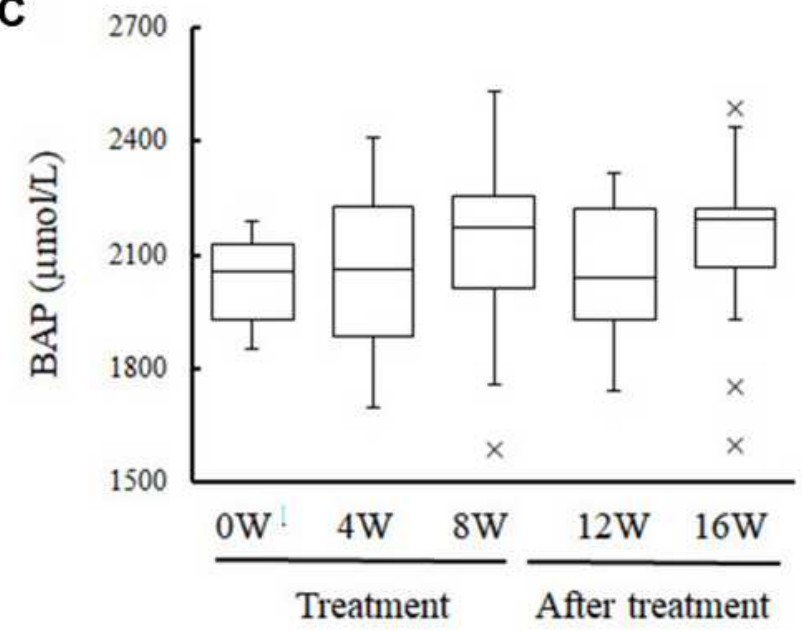

B

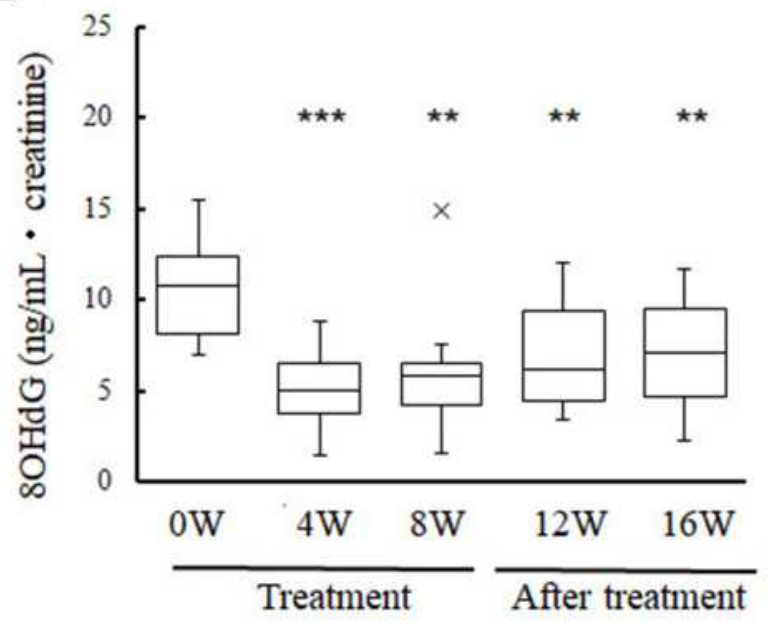

D

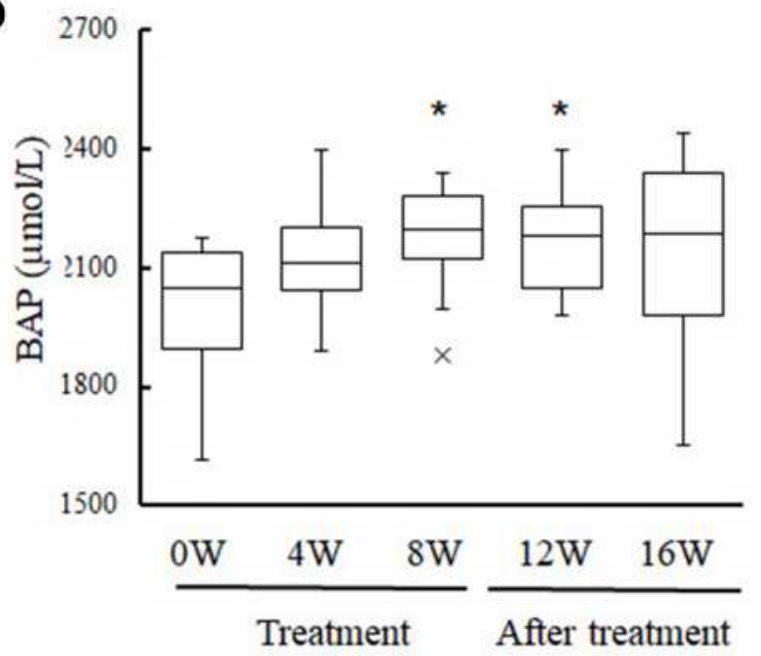

Figure 3 Comparison of findings before and after antioxidant treatment in patients with relatively high or low oxidative stress. (A) There was a significant increase in the 8-OHdG level at weeks 12 and 16 in patients with relatively low oxidative stress. (B) There was a significant decrease in 8-OHdG level at weeks 4, 8, 12 and I6 in patients with relatively high oxidative stress. (C) BAP level did not significantly change before and after treatment in patients with relatively low oxidative stress. (D) BAP level significantly increased at weeks 8 and 12 in patients with relatively high oxidative stress. ${ }^{*} p<0.05, * * p<0.01, * * * p<0.001$.

the group of NTG patients with relatively higher oxidative stress (Figure 3D). This might have been due to the participants improving their lifestyle after starting this study, potentially affecting our finding of a change in antioxidative stress levels after supplementation was stopped. Nevertheless, when we analyzed a group of patients with a higher BAP level than the overall average for our participants $(2022.37 \mu \mathrm{mol} / \mathrm{L})$, we found that their BAP level was not significantly elevated during supplement intake (data not shown). The BAP change we observed in the other groups was interesting. We speculate that the increased BAP level might return to its baseline with longer term observation. There is therefore a need for a longer follow-up study. Third, we observed a significant elevation in $8-\mathrm{OHdG}$ after supplementation for four and eight weeks, while we observed significant differences in 8-OHdG before and after supplementation in patients with relatively lower oxidative stress (Figure 3A). This discrepancy might have been due to an increase in oxidative stress after supplementation, causing increased endogenous antioxidant activity. Finally, we could not include a control group, because we could not find potential candidates without cataracts, due to the advanced age of our patients. We were also limited in our ability to detect relatively modest associations, making longer follow-up of the subjects necessary in a future study.

Our study indicates that patients with a low systemic antioxidant level may have increased susceptibility to oxidative stress, which might accelerate the progression of glaucoma. This raises the possibility that antioxidant 
supplementation may be a viable option to delay or prevent age-related glaucoma progression.

\section{Conclusion}

Oxidative stress may be a key non-IOP risk factor for agerelated cataracts and glaucoma. Our study revealed that antioxidant supplementation was effective in patients with high oxidative stress level, suggesting that such supplementation may be a novel way of combating diseases induced by systemic oxidative stress, and could contribute to individualized treatment for these diseases.

\section{Abbreviations}

ROS, reactive oxygen species; IOP, intraocular pressure; RGC, retinal ganglion cell; OAG, open-angle glaucoma; NTG, normal-tension glaucoma; BAP, biological antioxidant potential; MD, mean deviation; HFA, Humphrey field analyzer; 8-OHdG, 8-hydroxy-2'-deoxyguanosine; NMDA, N-methyl-D-aspartate.

\section{Data Sharing Statement}

The authors do not intend to share participant level data.

\section{Ethics Approval and Informed Consent}

This study adhered to the Declaration of Helsinki and Clinical Trials Act was approved by the Clinical Research Review Board of Tohoku University (study 2019-6-068), which is certified by the Japanese Ministry of Health, Labor and Welfare. The trial was registered with the UMIN clinical trial registry, number 000032050. Written informed consent was obtained from all subjects before the start of the study.

\section{Acknowledgments}

The authors thank Mr. Tim Hilts for editing the language of the manuscript, and Ms. Ruriko Igarashi for providing excellent technical support with oxidative stress measurements. We thank Dr. Hisayo Kubota, Dr. Hideyo Kudo, Dr. Megumi Uematsu, Dr. Marika Sato, and Dr. Naoko Takada for their medical treatment support. We thank Orthmedico Inc. for analyzing the data.

\section{Author Contributions}

All authors contributed to data analysis, drafting or revising the article, have agreed on the journal to which the article will be submitted, gave final approval of the version to be published, and agree to be accountable for all aspects or the work.

\section{Disclosure}

Professor Toru Nakazawa reports grants from Wakamoto Pharmaceutical Co., Ltd. during the conduct of this study; grants and personal fees from Santen Pharmaceutical Co., Ltd., Senju Pharmaceutical Co., Ltd., and Topcon Corporation, outside the submitted work; grants from Nidek Co., Ltd. This research was supported by Wakamoto Pharmaceutical Co., Ltd. The authors report no other conflicts of interest in this work.

\section{References}

1. Kaur J, Kukreja S, Kaur A, Malhotra N, Kaur R. The oxidative stress in cataract patients. J Clin Diagn Res. 2012;6(10):1629-1632. doi: 10.7860/JCDR/2012/4856.2626

2. Himori N, Kunikata H, Shiga Y, et al. The association between systemic oxidative stress and ocular blood flow in patients with normal-tension glaucoma. Graefes Arch Clin Exp Ophthalmol. 2016;254(2):333-341. doi:10.1007/s00417-015-3203-z

3. Cecilia OM, Jose Alberto CG, Jose NP, et al. Oxidative stress as the main target in diabetic retinopathy pathophysiology. J Diabetes Res. 2019;2019:8562408. doi:10.1155/2019/8562408

4. Pawlowska E, Szczepanska J, Koskela A, Kaarniranta K, Blasiak J. Dietary polyphenols in age-related macular degeneration: protection against oxidative stress and beyond. Oxid Med Cell Longev. 2019;2019:9682318. doi:10.1155/2019/9682318

5. Tezel G. Oxidative stress in glaucomatous neurodegeneration: mechanisms and consequences. Prog Retin Eye Res. 2006;25 (5):490-513. doi:10.1016/j.preteyeres.2006.07.003

6. Foster A, Resnikoff S. The impact of Vision 2020 on global blindness. Eye (Lond). 2005;19(10):1133-1135. doi:10.1038/sj. eye. 6701973

7. Guo Y, Chen X, Zhang H, et al. Association of OPA1 polymorphisms with NTG and HTG: a meta-analysis. PLoS One. 2012;7(8):e42387. doi:10.1371/journal.pone.0042387

8. Shiga Y, Kunikata H, Aizawa N, et al. Optic nerve head blood flow, as measured by laser speckle flowgraphy, is significantly reduced in preperimetric glaucoma. Curr Eye Res. 2016:1-7.

9. Inoue-Yanagimachi M, Himori N, Sato K, et al. Association between mitochondrial DNA damage and ocular blood flow in patients with glaucoma. Br J Ophthalmol. 2019;103(8):1060-1065. doi:10.1136/ bjophthalmol-2018-312356

10. Ferreira SM, Lerner SF, Brunzini R, Evelson PA, Llesuy SF. Oxidative stress markers in aqueous humor of glaucoma patients. Am J Ophthalmol. 2004;137(1):62-69. doi:10.1016/S0002-9394(03) 00788-8

11. Sacca SC, Pascotto A, Camicione P, Capris P, Izzotti A. Oxidative DNA damage in the human trabecular meshwork: clinical correlation in patients with primary open-angle glaucoma. Arch Ophthalmol. 2005;123(4):458-463. doi:10.1001/archopht.123.4.458

12. Feilchenfeld Z, Yucel YH, Gupta N. Oxidative injury to blood vessels and glia of the pre-laminar optic nerve head in human glaucoma. Exp Eye Res. 2008;87(5):409-414. doi:10.1016/j. exer.2008.07.011 
13. Nakazawa T. Ocular blood flow and influencing factors for glaucoma. Asia Pac J Ophthalmol (Phila). 2016;5(1):38-44. doi:10.1097/ APO.0000000000000183

14. Asano Y, Himori N, Kunikata H, et al. Age- and sex-dependency of the association between systemic antioxidant potential and glaucomatous damage. Sci Rep. 2017;7(1):8032. doi:10.1038/s41598-01708624-4

15. Uchida K, Himori N, Hashimoto K, et al. The association between oxidative stress and corneal hysteresis in patients with glaucoma. Sci Rep. 2020;10(1):545. doi:10.1038/s41598-020-57520-x

16. Harris A, Gross J, Moore N, et al. The effects of antioxidants on ocular blood flow in patients with glaucoma. Acta Ophthalmol. 2018;96(2):e237-e241. doi:10.1111/aos.13530

17. Park JW, Kwon HJ, Chung WS, Kim CY, Seong GJ. Short-term effects of Ginkgo biloba extract on peripapillary retinal blood flow in normal tension glaucoma. Korean $J$ Ophthalmol. 2011;25 (5):323-328. doi:10.3341/kjo.2011.25.5.323

18. Harada C, Noro T, Kimura A, et al. Suppression of oxidative stress as potential therapeutic approach for normal tension glaucoma. Antioxidants (Basel). 2020;9(9). doi:10.3390/antiox9090874

19. Maekawa S, Sato K, Fujita K, et al. The neuroprotective effect of hesperidin in NMDA-induced retinal injury acts by suppressing oxidative stress and excessive calpain activation. Sci Rep. 2017;7 (1):6885. doi:10.1038/s41598-017-06969-4

20. Escalona-Arranz JC, Perez-Roses R, Rodriguez-Amado J, et al. Antioxidant and toxicological evaluation of a Tamarindus indica L. leaf fluid extract. Nat Prod Res. 2016;30(4):456-459. doi:10.1080/14786419.2015.1019350

21. Natukunda S, Muyonga JH, Mukisa IM. Effect of tamarind (Tamarindus indica L.) seed on antioxidant activity, phytocompounds, physicochemical characteristics, and sensory acceptability of enriched cookies and mango juice. Food Sci Nutr. 2016;4 (4):494-507. doi:10.1002/fsn3.311

22. Komutarin T, Azadi S, Butterworth L, et al. Extract of the seed coat of Tamarindus indica inhibits nitric oxide production by murine macrophages in vitro and in vivo. Food Chem Toxicol. 2004;42 (4):649-658. doi:10.1016/j.fct.2003.12.001
23. Agnihotri A, Singh V. Effect of Tamarindus indica Linn. and Cassia fistula Linn. stem bark extracts on oxidative stress and diabetic conditions. Acta Pol Pharm. 2013;70(6):1011-1019.

24. Martinello F, Soares SM, Franco JJ, et al. Hypolipemic and antioxidant activities from Tamarindus indica L. pulp fruit extract in hypercholesterolemic hamsters. Food Chem Toxicol. 2006;44 (6):810-818. doi: $10.1016 / j$.fct.2005.10.011

25. Ohba T, Ishisaka M, Tsujii S, et al. Crocetin protects ultraviolet A-induced oxidative stress and cell death in skin in vitro and in vivo. Eur JPharmacol. 2016;789:244-253. doi:10.1016/j.ejphar.2016.07.036

26. Nam KN, Park YM, Jung HJ, et al. Anti-inflammatory effects of crocin and crocetin in rat brain microglial cells. Eur J Pharmacol. 2010;648(1-3):110-116. doi:10.1016/j.ejphar.2010.09.003

27. Ohno Y, Nakanishi T, Umigai N, Tsuruma K, Shimazawa M, Hara H. Oral administration of crocetin prevents inner retinal damage induced by N-methyl-D-aspartate in mice. Eur J Pharmacol. 2012;690(1-3):84-89. doi:10.1016/j.ejphar.2012.06.035

28. Seifried HE, Anderson DE, Milner JA, Greenwald P. Reactive oxygen speicies and dietary antioxidants: double-edgedswords? In: Panglossi H, editor. New Developments in Antioxidant Research. Nova Science Publishers Inc; 2006:1-25.

29. Yang X, Hondur G, Tezel G. Antioxidant treatment limits neuroinflammation in experimental glaucoma. Invest Ophthalmol Vis Sci. 2016;57(4):2344-2354. doi:10.1167/iovs.16-19153

30. Caprioli J, Munemasa Y, Kwong JM, Piri N. Overexpression of thioredoxins 1 and 2 increases retinal ganglion cell survival after pharmacologically induced oxidative stress, optic nerve transection, and in experimental glaucoma. Trans Am Ophthalmol Soc. 2009; 107:161-165.

31. Kang JH, Willett WC, Rosner BA, Buys E, Wiggs JL, Pasquale LR. Association of Dietary nitrate intake with primary open-angle glaucoma: a prospective analysis from the nurses' Health Study and Health Professionals Follow-up Study. JAMA Ophthalmol. 2016;134 (3):294-303. doi:10.1001/jamaophthalmol.2015.5601

32. Lee J, Sohn SW, Kee C. Effect of Ginkgo biloba extract on visual field progression in normal tension glaucoma. J Glaucoma. 2013;22 (9):780-784. doi:10.1097/IJG.0b013e3182595075
Clinical Ophthalmology

\section{Publish your work in this journal}

Clinical Ophthalmology is an international, peer-reviewed journal covering all subspecialties within ophthalmology. Key topics include: Optometry; Visual science; Pharmacology and drug therapy in eye diseases; Basic Sciences; Primary and Secondary eye care; Patient Safety and Quality of Care Improvements. This journal is indexed on PubMed
Central and CAS, and is the official journal of The Society of Clinical Ophthalmology (SCO). The manuscript management system is completely online and includes a very quick and fair peer-review system, which is all easy to use. Visit http://www.dovepress.com/ testimonials.php to read real quotes from published authors.

\section{Dovepress}

